\title{
Extending the limits of globule detection $\star$
}

\section{ISOPHOT Serendipity Survey observations of interstellar clouds II}

\author{
L. V. Tóth ${ }^{1,2,3}$, Cs. Kiss ${ }^{4}$, M. Juvela ${ }^{1}$, M. Stickel ${ }^{3}$, U. Lisenfeld ${ }^{5}$, and S. Hotzel ${ }^{3}$ \\ 1 Helsinki University Observatory, PO Box 14, 00014 University of Helsinki, Finland \\ 2 Department of Astronomy, Loránd Eötvös University, Pázmány Péter sétány 1/a, 1117 Budapest, Hungary \\ 3 Max-Planck-Institut für Astronomie, Königstuhl 17, 69117 Heidelberg, Germany \\ 4 Konkoly Observatory, Budapest, PO Box 67, 1525, Hungary \\ 5 IRAM, Avenida Divina Pastora 7, N.C., 18012 Granada, Spain
}

Received 1 March 2002 / Accepted 2 September 2002

\begin{abstract}
Small isolated clouds were discovered by the ISOPHOT $170 \mu \mathrm{m}$ Serendipity Survey (ISOSS) as faint $I_{170}<$ $13 \mathrm{MJy} \mathrm{sr}^{-1}$ FIR sources. One of them is ISOSS J20246+6540, a cold $\left(T_{\mathrm{d}} \approx 14.5 \mathrm{~K}\right)$ pointlike ISOSS source without an IRAS pointsource counterpart. In the Digitized Sky Survey $B$ band it is seen as a 3'size bipolar nebulosity with an average excess DSS blue band surface brightness of $8 \%$ of the background's photographical density. The CO column density distribution determined by multi-isotopic, multi-level CO measurements with the IRAM-30 m telescope agrees well with the optical appearance. An average hydrogen column density of $\approx 10^{21} \mathrm{~cm}^{-2}$ was derived from both the FIR and CO data. Using a kinematic distance estimate of $400 \mathrm{pc}$ the NLTE modelling of the $\mathrm{CO}, \mathrm{HCO}^{+}$, and $\mathrm{CS}$ measurements gives a peak density of $\approx 10^{4} \mathrm{~cm}^{-3}$. The multiwavelength data characterise ISOSS $20246+6540$ as a representative of a class of globules which has not been discovered so far due to their small angular size and low $100 \mu \mathrm{m}$ brightness. A significant overabundance of ${ }^{13} \mathrm{CO}$ is found $X\left({ }^{13} \mathrm{CO}\right) \geq 150 \times X\left(\mathrm{C}^{18} \mathrm{O}\right)$. This is likely due to isotope selective chemical processes.
\end{abstract}

Key words. ISM: clouds - dust, extinction - ISM: molecules - Infrared: ISM: - Surveys

\section{Introduction}

Bok globules were originally detected in absorption against HII regions by Bok \& Reilly (1947), and are known as small, dense interstellar clouds in the solar neighbourhod ( $d \lesssim 400 \mathrm{pc}$ ). They were identified optically, and mostly the nearby ones have been catalogued so far. It is expected that they are similarily common elsewhere in the Galactic disk and in fact a few distant ones have already been investigated e.g. by Launhardt \& Henning (1997). Their FIR properties were determined by Clemens \& Barvainis (1988) based on IRAS data. They emphasized the importance of finding FIR faint cold globules since these are representatives of the inactive (i.e. non-starforming) interstellar medium. Starless globules with small apparent size and low temperature (thus also very low $100 \mu \mathrm{m}$ brightness) can be seen only by good sensitivity at wavelengths over $100 \mu \mathrm{m}$. An ISOPHOT study of pre-stellar cores in dark clouds with low $100 \mu \mathrm{m}$ brightness was recently

Send offprint requests to: L. V. Tóth,

e-mail: lvtoth@mpia-hd.mpg.de

* Based on observations with ISO, an ESA project with instruments funded by ESA Member States (especially the PI countries: France, Germany, The Netherlands and the UK) and with the participation of ISAS and NASA. reported by Ward-Thompson et al. (2002). The ISOPHOT $170 \mu \mathrm{m}$ Serendipity Survey (ISOSS) (Bogun et al. 1996) can be used to locate cold galactic objects even without any preliminary identification. This raises the possibility of detection of the "missing" globules via ISOSS. Our preceeding papers on ISOSS observations of interstellar clouds discussed cold cores in the well known Chamaeleon cloud complex (Tóth et al. 2000) and in the known B 68 globule (Hotzel 2001). We present our results on ISOSS J20246+6540 proving that it is, a small and cold isolated molecular cloud - one of the so far missed population.

\section{Observation and data analysis}

Far-infrared data: we searched for faint, small, cold sources with no IRAS associations in the region $95^{\circ}<l<115^{\circ}$, $10^{\circ}<b<20^{\circ}$, where the ISOSS survey has over $50 \%$ coverage. ISOSS $170 \mu \mathrm{m}$ data were smoothed to the IRAS ISSA (Wheelock et al. 1994) resolution, and processed according to Tóth et al. (2000) and Hotzel (2001). Sources were selected using the following criteria: a size $F W H M \leq 6^{\prime}$, a $170 \mu \mathrm{m}$ source surface brightness $8 \pm 5 \mathrm{MJy} \mathrm{sr}^{-1}$, and a background corrected colour parameter $C P=6.4 \pm 0.5$, no associations in the IRAS Point Source Catalogue, or in the IRAS 
Table 1. Five ISOSS globules and their nearest cloud neighbours, CB88: Clemens \& Barvainis (1988), YDM97: Yonekura (1997) (*) refers to an unnumbered peak, LM99: Lee \& Myers (1999).

\begin{tabular}{lrccl}
\hline \hline ISOSS name & $\begin{array}{r}b \\
{\left[{ }^{\circ}\right]}\end{array}$ & $\begin{array}{c}a \times b \\
{\left[{ }^{\circ}\right]}\end{array}$ & $\begin{array}{l}\text { nearest } \\
{\left[{ }^{\prime}\right]}\end{array}$ & neighbour \\
\hline ISOSS J20180+6345 & 97.71 & 15.29 & $5 \times 3$ & $8^{\prime} \mathrm{CB} 88$ \#219 \\
ISOSS J20215+6820 & 102.00 & 17.34 & $3 \times 2$ & $45^{\prime} \mathrm{YDM} 97$ \#4 \\
ISOSS J20246+6540 & 99.79 & 15.69 & $4 \times 2$ & $20^{\prime} \mathrm{YDM97(*)}$ \\
ISOSS J20380+6352 & 99.05 & 13.51 & $5 \times 2$ & $12^{\prime} \mathrm{CB} 88$ \#224 \\
ISOSS J20474+6014 & 96.72 & 10.46 & $5 \times 5$ & $14^{\prime} \mathrm{LM99} \mathrm{\# 349}$ \\
\hline
\end{tabular}

Faint Source Catalogue within $5^{\prime}$, and no excess in the IRAS ISSA maps above $0.6 \mathrm{MJy} \mathrm{sr}^{-1}$. We located 50 objects fullfilling our criteria. ISOSS J20246+6540 was also detected as an $I_{170}($ peak $)=4 \mathrm{MJy} \mathrm{sr}^{-1}(>4 \sigma)$ pointlike source at $\mathrm{RA}(2000)=$ $20^{\mathrm{h}} 24^{\mathrm{m}} 36^{\mathrm{s}} \operatorname{Dec}(2000)=+65^{\circ} 40^{\prime}\left(l=99.8^{\circ}, b=15.7^{\circ}\right)$. with an unsmoothed $F W H M \leq 150^{\prime \prime}$. We note that the unsmoothed ISOSS point-spread function is nearly Gaussian with $F W H M \approx 130^{\prime \prime}$.

Optical data: the Digital Sky Survey (DSS2) blue plates indicated optical counterparts for 41 of the 50 ISOSS sources, out of which 5 isolated, globule like sources were found. Three of them bear opaque cores resembling the globule of Dickman $\&$ Clemens (1983) and two are without any opaque core: ISOSS J20215+6820 and ISOSS J20246+6540. Table 1 lists (1) ISOSS names, $(2,3)$ galactic coordinates, (4) optical dimensions in arcminutes, (5) the distance to the nearest cloud neighbours

Further 8 sources were associated with complexes of reflecting clouds. Detailed optical study of the 5 globule like clouds and a study of statistical issues will be given elsewhere. As an example we present results on ISOSS J20246+6540 which was studied using DSS2 blue and red images. In order to make surface brightness maps of ISOSS J20246+6540, stars were removed from the optical images by substituting the average surrounding photographical density value.

Radio spectroscopic measurements: the $J=(1-0)$ and (2-1) rotational lines of $\mathrm{CO},{ }^{13} \mathrm{CO}$ and $\mathrm{C}^{18} \mathrm{O}$ were measured in Aug. 1998 with the IRAM-30 m telescope. A $4.5^{\prime} \times 4.5^{\prime}$ region centered on ISOSS $20246+6540$ was mapped in on-thefly mode in ${ }^{12} \mathrm{CO}(1-0),{ }^{12} \mathrm{CO}(2-1)$ and ${ }^{13} \mathrm{CO}(1-0)$ with angular resolutions of $22^{\prime \prime}, 11^{\prime \prime}, 22^{\prime \prime}$ respectively. Pointed measurements were performed in all the above mentioned transitions at the ${ }^{13} \mathrm{CO}(1-0)$ peak position $\left(22^{\mathrm{h}} 24^{\mathrm{m}} 46^{\mathrm{s}}, 65^{\circ} 40^{\prime} 01^{\prime \prime}\right)$. Spectral resolutions of $0.1,0.05$ and $0.03 \mathrm{~km} \mathrm{~s}^{-1}$ were used. These spectra are shown in Fig. 1. The lines are narrow with a FWHM of $\leq 0.5 \mathrm{~km} \mathrm{~s}^{-1}$. The results of the $1998 \mathrm{C}^{18} \mathrm{O}$ pointed measurements were confirmed in Sep. 2000 when also the CS(2-1) and (3-2) as well as the $\mathrm{HCO}^{+}(1-0)$ and (2-1) transitions were observed with the IRAM-30 telescope. The $\mathrm{C}^{18} \mathrm{O}, \mathrm{CS}(2-1)$ and $\mathrm{HCO}^{+}(1-0)$ lines were detected with $S / N>5$ and well resolved with the $0.03 \mathrm{~km} \mathrm{~s}^{-1}$ spectral channels. The data were calibrated to the scale of corrected antenna temperature, $T_{\mathrm{A}}^{*}$, by observing loads at ambient and cold temperature, as in the
Table 2. Radio spectroscopy results for pointed measurements towards the NE lobe, $\Delta T_{\text {rms }}$ is given in parentheses after $T_{\mathrm{MB}}$, errors of the Gaussian line fit are given in parentheses for $F W H M$ and $v_{\mathrm{LSR}}$, asterisk marks selfabsorbed lines.

\begin{tabular}{llll}
\hline \hline line & $\begin{array}{l}T_{\mathrm{MB}} \\
{[\mathrm{K}]}\end{array}$ & $\begin{array}{l}F W H M \\
{\left[\mathrm{~km} \mathrm{~s}^{-1}\right]}\end{array}$ & $\begin{array}{l}v_{\mathrm{LSR}} \\
{\left[\mathrm{km} \mathrm{s}^{-1}\right]}\end{array}$ \\
\hline${ }^{12} \mathrm{CO}(1-0)$ & $6.7^{*}(0.11)$ & $0.43(0.01)$ & $-2.70(0.01)$ \\
${ }^{12} \mathrm{CO}(2-1)$ & $5.41(0.17)$ & $0.47(0.01)$ & $-2.69(0.01)$ \\
${ }^{13} \mathrm{CO}(1-0)$ & $4.3^{*}(0.14)$ & $0.32(0.03)$ & $-2.68(0.01)$ \\
${ }^{13} \mathrm{CO}(2-1)$ & $3.60(0.08)$ & $0.31(0.01)$ & $-2.70(0.01)$ \\
$\mathrm{C}^{18} \mathrm{O}(1-0)$ & $0.11(0.02)$ & $0.25(0.02)$ & $-2.68(0.01)$ \\
$\mathrm{C}^{18} \mathrm{O}(2-1)$ & $0.10(0.03)$ & $0.24(0.03)$ & $-2.76(0.02)$ \\
$\mathrm{CS}^{2}(2-1)$ & $0.09(0.02)$ & $0.25(0.02)$ & $-2.69(0.01)$ \\
$\mathrm{HCO}^{+}(1-0)$ & $0.20(0.03)$ & $0.29(0.03)$ & $-2.70(0.01)$ \\
\hline
\end{tabular}

conventional "chopper-wheel" calibration for millimetre wavelength observations. The data were converted to the main-beam scale by applying the relation $T_{\mathrm{MB}}=T_{\mathrm{A}}^{*}\left(F_{\text {eff }} / B_{\text {eff }}\right)$. The values for the main-beam efficiency, $B_{\text {eff }}$, are 0.70 and 0.42 (July 1998), and 0.75 and 0.53 (September 2000), and the values for the forward efficiency, $F_{\text {eff }}$, are 0.92 and 0.85 for $\mathrm{CO}(1-0)$ and $\mathrm{CO}(2-1)$, respectively. We checked the calibration by observing the standard sources DR 21 and $\mathrm{W} 3 \mathrm{OH}$ and found it always to be better than $20 \%$. Results are listed in Table 2 .

\section{Results}

\subsection{FIR results and derived parameters}

ISOSS J20246+6540 is a faint FIR source with an upper limit on the $I_{100}$ brightness of about $0.5 \mathrm{MJy} \mathrm{sr}^{-1}$ derived from the "raw" (IRDS format) IRAS data. Comparison of ISOSS and ISSA data (Wheelock et al. 1994) was made as described by Tóth et al. (2000). An upper limit of the colour temperature of $14.5 \mathrm{~K}$ was estimated from the bisector slope of the $I_{170}$ vs. $I_{100}$ scatter plot. Assuming a dust temperature of $14.5 \mathrm{~K}$, an average dust column density of $1.3 \times 10^{-5} \mathrm{~g} \mathrm{~cm}^{-2}$ was derived following Hildebrand (1983). This corresponds to an average hydrogen column density of $N\left(\mathrm{HI}+2 \mathrm{H}_{2}\right)=8.6 \times 10^{20} \mathrm{~cm}^{-2}$ where a hydrogen-to-dust mass ratio of 110 (Launhardt \& Henning 1997) was assumed.

\subsection{Optical images}

ISOSS J20246+6540 appears as a faint, isolated reflection cloud west of the L1155/L1157 cloud complex (Lynds 1962). When smoothed to $15^{\prime \prime}$ the excess diffuse surface brightness distribution of ISOSS 20246+6540 shows a "bright" lobe at the NE and a fainter fragmented one at SE. The $\sigma_{\mathrm{B}}$ standard deviation of photographical density outside the globule on the star-removed, smoothed image was $1.3 \%$ of the average background value in the blue band image. The POSS blue band diffuse surface brightness is shown in Fig. 2a, where the lowest contour is drawn at an excess of three times $\sigma_{\mathrm{B}}$. The average excess surface brightness of ISOSS J20246+6540 is $\approx 8 \%$ of the background photographic density value in the DSS blue band. The same morphology is shown by the $B$ and $R$ surface brightness distributions, except that the SE lobe is slightly more 

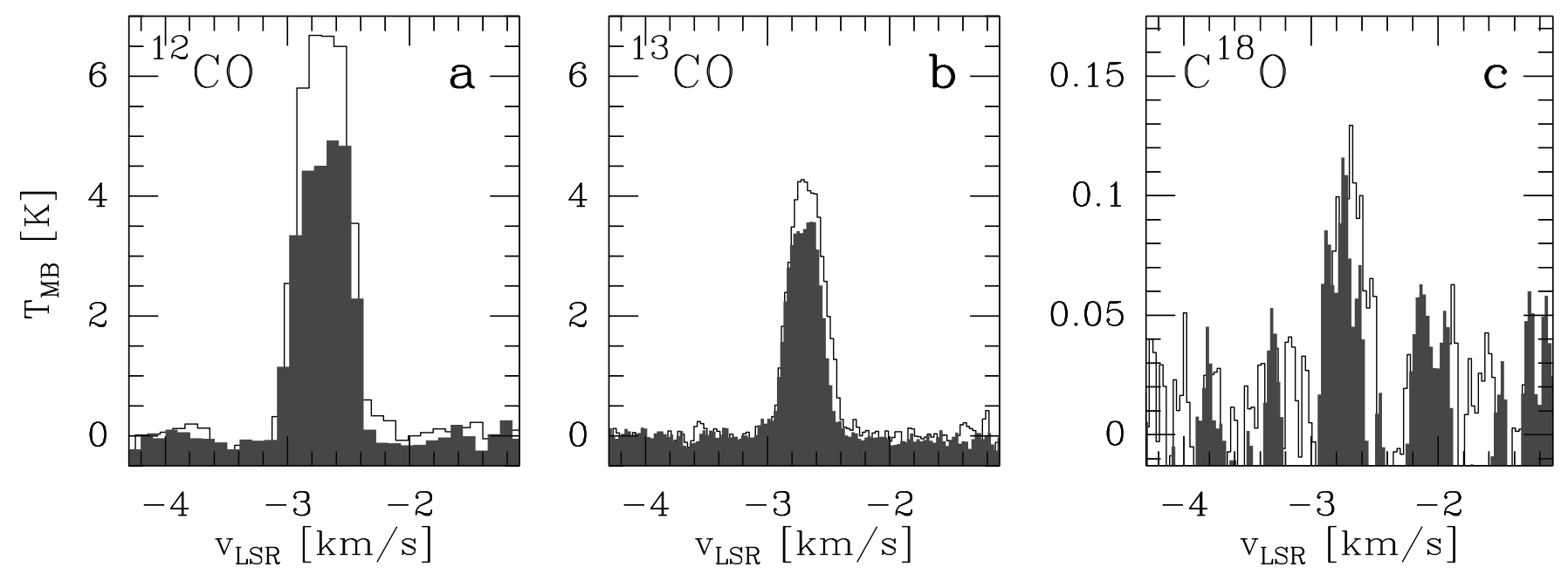

Fig. 1. CO spectra at the ${ }^{13} \mathrm{CO}(1-0)$ peak, $(2-1)$ lines are overlaid as filled histograms. a) ${ }^{12} \mathrm{CO}(1-0)$ and $(2-1) \operatorname{spectra}$. b) ${ }^{13} \mathrm{CO}(1-0)$ and $(2-1)$ spectra. c) $\mathrm{C}^{18} \mathrm{O}(1-0)$ and (2-1) spectra.
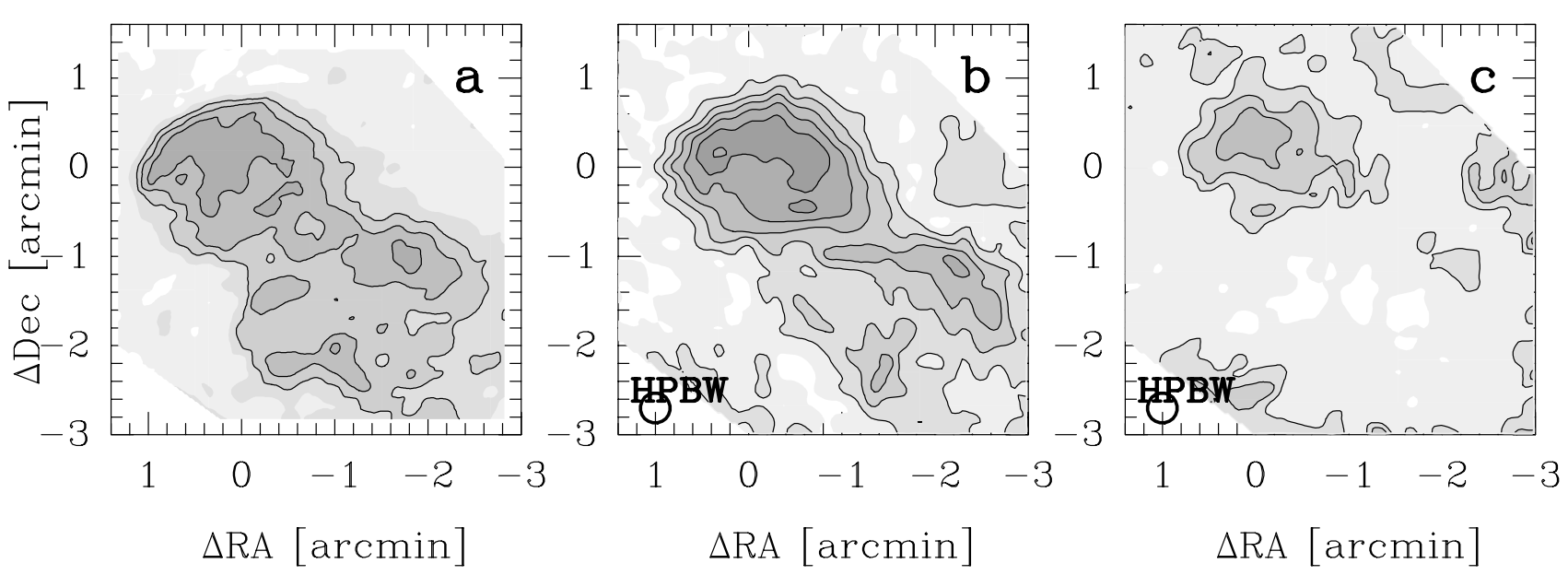

Fig. 2. Dust and gas in ISOSS 20246+6540, reference coordinate $(0,0):$ RA $=20^{\mathrm{h}} 24^{\mathrm{m}} 44^{\mathrm{s}}$ Dec $=65^{\circ} 40^{\prime} 04^{\prime \prime}$. a) DSS-2 blue band diffuse surface brightness, contours are drawn at photograpic density values of 4,8 and $12 \%$ above the background level. b) $W\left({ }^{12} \mathrm{CO}(1-0)\right)$, contours are from $1.5 \mathrm{~K} \mathrm{~km} \mathrm{~s}^{-1}$ with $1.5 \mathrm{~K} \mathrm{~km} \mathrm{~s}^{-1}$ steps; c) ${ }^{13} \mathrm{CO}(1-0)$, contours are from $1.0 \mathrm{~K} \mathrm{~km} \mathrm{~s}^{-1}$ with $1.0 \mathrm{~K} \mathrm{~km} \mathrm{~s}^{-1}$ steps; CO isotopomer line intensities are integrated in the velocity interval $-5 \mathrm{~km} \mathrm{~s}^{-1} \leq v_{\mathrm{LSR}} \leq 0 \mathrm{~km} \mathrm{~s}^{-1}$.

red than the NW lobe. The lack of a dark core indicates an average visual extinction below $1.5 \mathrm{mag}$.

\subsection{Molecular line results, and derived parameters}

All observed lines show a LSR velocity of $-2.7 \mathrm{~km} \mathrm{~s}^{-1}$. The FWHM widths of the ${ }^{12} \mathrm{CO}$ lines are around $0.4 \mathrm{~km} \mathrm{~s}^{-1}$, and around $0.3 \mathrm{~km} \mathrm{~s}^{-1}$ for all the other detected lines. The ${ }^{12} \mathrm{CO}$ and ${ }^{13} \mathrm{CO}$ integrated intensity distributions are shown in Figs. $2 \mathrm{~b}$ and c. The bipolar shape is well seen and the CO line intensities are in accordance with the excess surface brightness distribution, the NE lobe being much brighter. The two lobes show the same radial velocity.

The ${ }^{12} \mathrm{CO}$ and ${ }^{13} \mathrm{CO}$ lines trace the ISM well over most of the cloud. A comparison of $\mathrm{C}^{18} \mathrm{O}$ to ${ }^{13} \mathrm{CO}$ lines (both $1-0$ and $2-1)$ at the centre of the NE lobe indicates an underabundance of $\mathrm{C}^{18} \mathrm{O}$ by a factor of 4 . This effect is expected in cold clouds with moderate density, exposed to UV radiation (Glassgold et al. 1985). A more careful modelling may account for it as we show in Sect. 4.2.

\section{Discussion}

\subsection{Distance of ISOSS J20246+6540}

The distance of ISOSS $20246+6540$ can be estimated, relating it to its neighbours. Its nearest neighbours are L1122 (Lynds 1962), and the YDM97 CO1 (Yonekura 1997). The ${ }^{13} \mathrm{CO}$ survey of Cepheus by Yonekura (1997) covers the position of ISOSS J20246+6540 and their Fig. 6a indicates a few small clouds around ISOSS J20246+6540 (i.e. YDM97 CO1, YDM97 CO2, YDM97 CO3) and one even smaller unnumbered peak very close to ISOSS J20246+6540 at $l=100.1 b=$ 15.7. All the listed Yonekura clouds have $v_{\mathrm{LSR}}>+2.5 \mathrm{~km} \mathrm{~s}^{-1}$, 
and they are counted into the "close group" of clouds, which on the other hand is associated with extended FIR features around ISOSS J20246+6540. The nearest molecular clouds with negative $v_{\mathrm{LSR}}$ are YDM97 CO7, CO9, CO10 at $l \approx 103^{\circ} .0 b \approx 16^{\circ} .7$. ISOSS $20246+6540$ itself has $v_{\mathrm{LSR}}=-2.7 \mathrm{~km} \mathrm{~s}^{-1}$. It probably belongs to one of the ISM layers of the nearby Cepheus Flare GMC, and is located at about $400 \mathrm{pc}$ (Kun 1998). We note that applying the size-linewidth relation of Larson (1981) the globule may be between 100 and 400 pc.

\subsection{Radiative transfer models}

We have modelled the NE lobe of the bipolar globule with spherically symmetric cloud models, although the NE clump shows some deviations from spherical symmetry in both ${ }^{12} \mathrm{CO}$ and ${ }^{13} \mathrm{CO}$ (see Figs. $2 \mathrm{~b}$ and c). With RA $=20^{\mathrm{h}} 24^{\mathrm{m}} 44^{\mathrm{s}}$ Dec $=+65^{\circ} 40^{\prime} 04^{\prime \prime}$ as the centre position, we have averaged spectra in concentric rings with radii increasing by $10^{\prime \prime}$ intervals up to a radius of $90^{\prime \prime}$. The effective resolution of the averaged spectra is $40^{\prime \prime}$ for the $J=1-0$ lines and $20^{\prime \prime}$ for the $J=2-1$ lines.

We set the cloud parameters as follows.

(1) We assume a density distribution $n \sim r^{-1.5}$ with a density ratio 20 between the centre and the cloud surface.

(2) The kinetic temperature is assumed to rise linearly from the cloud centre. This is a crude approximation of the actual temperature structure of for a small, spherically symmetric globule without internal heating sources (e.g. Leung 1985; Nelson \& Langer 1999) but will suffice for the present purposes. The temperature gradient, i.e. the difference between the outermost and innermost shells $\Delta T=0 \mathrm{~K}, 6 \mathrm{~K}$ or $10 \mathrm{~K}$. Higher contrast than $10 \mathrm{~K}$ means too high a temperature for the outer cloud, in contradiction with the observed small linewidth.

(3) Extinction-dependent relative molecular abundances $X($ molecule $)=\frac{n \text { (molecule })}{n(H)}$ were estimated according to Warin et al. (1996). The cloud is cold, exposed to UV radiation and it has a peak visual extinction between 1 and 2 mag. In these conditions isotope selective processes result in a relative overabundance of ${ }^{13} \mathrm{CO}$ and relative underabundance of $\mathrm{C}^{18} \mathrm{O}$ according to Bally \& Langer (1982). When applying the Warin et al. (1996) relative abundances, we introduced an intrinsic extinction at the cloud boundary since the ${ }^{12} \mathrm{CO}$ lines are not vanishing at the boundary of the NE lobe. This assumption is supported by the presence of surrounding extended cirrus-like emission seen at $100 \mu \mathrm{m}$ on the ISSA image.

(4). Distance: 100, 200, 400, 600, 800, 1000, and 2000 pc were tested.

When the density, temperature, relative abundance distributions and the distance are set to a value allowed by the above constrains, the free model parameters are the central density $\left(n_{\mathrm{c}}\right)$, the intrinsic linewidth $(\Delta v)$ and the angular diameter $(D)$ of the model cloud. The radiative transfer problem is solved with Monte Carlo simulation (Juvela 1997). The computed spectra are convolved to the resolution of the observed spectra and the quality of the fit between the two is estimated with a weighted $\chi^{2}$ value. The model cloud is divided into 31 shells of equal thickness and the free parameters are optimized
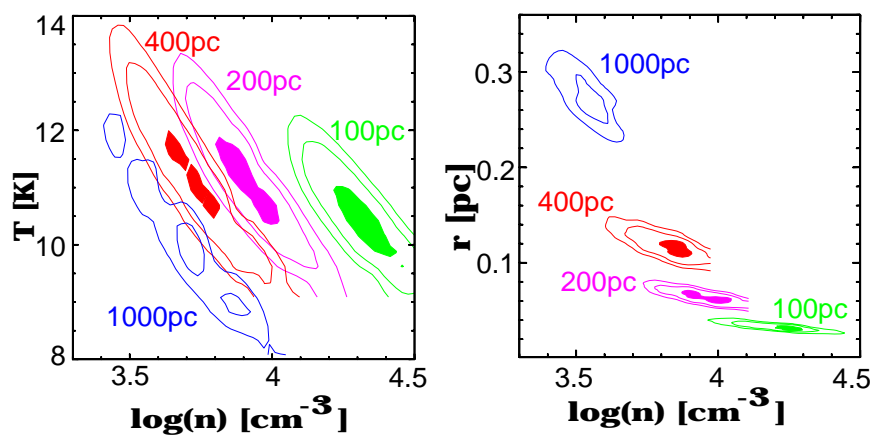

Fig. 3. Results of the radiative transfer modelling of the northern lobe assuming distances: 100 pc (yellow), 200 pc (lilac), 400 pc (red), $1000 \mathrm{pc}$ (blue). The relative $\chi^{2}$ minimum regions are plotted as shaded regions in selected planes of the parameter space. Two further contours of the relative $\chi^{2}$ of $1.14 \& 1.29$ are overlaid. (Extinction dependent relative molecular abundances were used.) a) Density versus kinetic temperature (left); b) density versus size (right).

separately for ${ }^{12} \mathrm{CO}$ and ${ }^{13} \mathrm{CO}$. We then select the set of parameters which provides the best fits for both. Since the ${ }^{12} \mathrm{CO}$ observations only probe the outer layers of the cloud, the ${ }^{12} \mathrm{CO}$ based estimate of the column density is uncertain. Modelling based on the ${ }^{13} \mathrm{CO}$ line, however, gives surprisingly similar results when the appropriate average relative abundance value $X\left({ }^{13} \mathrm{CO}\right) \approx 1.1 \times 10^{-6}$ is selected. The models are not sensitive to $20 \%$ changes in the average molecular abundances or density, although similar changes of the kinetic temperature or size are, critical (see Fig. 3).

Synthetic spectra for $\mathrm{C}^{18} \mathrm{O} \mathrm{CS}$ and $\mathrm{HCO}^{+}$were generated with the NLTE model using the best parameter sets (lowest $\chi^{2}$ ) from the ${ }^{12} \mathrm{CO}{ }^{13} \mathrm{CO}$ analysis. The relative abundances were varied up to $100 \%$ and the other parameters up to $30 \%$. The $\mathrm{C}^{18} \mathrm{O}$ lines were best reproduced assuming an average relative abundance of $X\left({ }^{13} \mathrm{CO}\right) / X\left(\mathrm{C}^{18} \mathrm{O}\right) \approx 150$, an extreme but possible underabundance by factor of 28 (Glassgold et al. 1985). The pointed measurements supported the density and temperature results shown in Fig. 3. The derived NLTE kinetic temperature is around $11 \mathrm{~K}$ and assuming a distance of $400 \mathrm{pc}$ the peak hydrogen density and the size of the NE lobe are $n_{\mathrm{c}}=6.7 \times 10^{4} \mathrm{~cm}^{-3}$ and $0.12 \mathrm{pc}$ respectively. The column density estimate is $N\left(\mathrm{H}_{2}\right) \approx 2 \times 10^{21} \mathrm{~cm}^{-2}$. This result is in agreement with the column density derived from the FIR data. The total gas mass would be $\approx 3 M_{\odot}$.

With $N\left(\mathrm{H}_{2}\right) \sim 10^{21} \mathrm{~cm}^{-2}$, the visual extinction towards the cloud centre is $A_{\mathrm{V}} \approx 1$ (Bohlin et al. 1978) and the cloud is optically thick for UV photons unless it is very clumpy. External heating, however, is reduced by the surrounding ISM, which is represented by the nonvanishing ${ }^{12} \mathrm{CO}$ lines. This may be the reason that a moderate $6 \mathrm{~K}$ temperature contrast was found to be more likely than a $10 \mathrm{~K}$ contrast. A similar temperature profile was found by Ciardi et al. (2000) in one of the dense cloud cores of L 1082 .

Although the SW clump is clearly elongated similar modelling was carried out for that part of the cloud. Observed spectra were averaged over annuli at radii up to $50^{\prime \prime}$ from the clump centre. Assuming a model where the kinetic temperature 
increases linearly from the centre, we obtain a peak column density of $N\left(\mathrm{H}_{2}\right)=2.9 \times 10^{20} \mathrm{~cm}^{-2}$ based on the CO spectra.

\subsection{Frequency of faint globules}

In the Yonekura (1997) data ${ }^{13} \mathrm{CO}$ spectra were found for one of the 5 clouds. ISOSS J20215+6820 appeared as a small $\left(F W H M \leq 5^{\prime}\right)$ isolated molecular cloud with $M \approx 2 M_{\odot}$ when $T_{\mathrm{ex}}=11 \mathrm{~K}$ and a distance of $d=400 \mathrm{pc}$ was assumed (Yonekura 2002). The 2 ISOSS sources without opaque cores have their nearest cloud neighbours outside a $15^{\prime}$ search radius. The 3 opaque cloudlets are separated from their companions by about $10^{\prime}$ (see also Table 1).

All the 5 globule like sources were found inside the fainter half of the studied region, i.e. in $95^{\circ}<l<105^{\circ}, 10^{\circ}<b<20^{\circ}$, where the ISOSS slew coverage was above $70 \%$, and the average $170 \mu \mathrm{m}$ sky brightness is $\approx 30 \mathrm{MJy} \mathrm{sr}^{-1}$. This kind of sources may be similarly common in other galactic regions. However detecting them by ISOSS may be more difficult at regions with higher FIR background/foreground brightness values.

\section{Concluding remarks}

1. ISOSS $20246+6540$ is a small isolated molecular cloud discovered by its $170 \mu \mathrm{m}$ emission i the unbiased ISO Serendipity Survey.

2. The globule appears as bipolar in both optical surface brightness and mm-line maps.

3. A distance of $400 \mathrm{pc}$ is likely, setting the diameter of the northern lobe to approximately $0.1 \mathrm{pc}$.

4. The average physical parameters are: $T_{\text {kin }}=11 \mathrm{~K}, n\left(\mathrm{H}_{2}\right)=$ $7000 \mathrm{~cm}^{-3}$.

5. The population of small and faint starless globules can only be effectively explored by high sensitivity FIR measurements such as those carried out by PHT-C200 on board ISO.

6. Our finding demonstrates the importance of such unbiased surveys by future missions like SIRTF, ASTRO-F or Planck.

Acknowledgements. We acknowledge the numerous valuable comments by Kalevi Mattila, suggestions by an anonymous referee, and important notes by Mark Rawlings. The ISOPHOT project and Postoperation Phase was funded by the Deutsche Agentur für Raumfahrtangelegenheiten (DARA, now DLR), the Max-PlanckGesellschaft, the Danish, British and Spanish Space Agencies and several European and American institutes.
Members of the Consortium on the ISOPHOT Serendipity Survey (CISS) are MPIA Heidelberg, ESA ISO SOC Villafranca, AIP Potsdam, IPAC Pasadena, Imperial College, London.

This research was partly supported by the OTKA F-022566 grant and by the Academy of Finland grants Nos. 158300, 173727, 174854 and by "The Jenny and Antti Wihuri Foundation".

This research has made use of the Digitized Sky Survey, produced at the Space Telescope Science Institute, NASA's Astrophysics Data System Abstract Service, the Simbad Database, operated at CDS, Strasbourg, France.

\section{References}

Bally, J., \& Langer, W. D. 1982, ApJ, 255, 143

Bogun, S., Lemke, D., Klaas, U., et al. 1996, A\&A, 315, L71

Bohlin, R. C., Savage, B. D., \& Drake, J. F. 1978, ApJ, 224, 132

Bok, B. J., \& Reilly, E. F. 1947, ApJ, 105, 255

Ciardi, D. R., Woodward, C. E., Clemens, D. P., et al. 2000, AJ, 120, 393

Clemens, D. P., \& Barvainis, R. 1988, ApJS, 68, 257

Cutri, R. M., Low, F. J., \& Guhathrakurta, P. 1993, PASP, 105, 106

Dickman, R. L., \& Clemens, D. P. 1983, ApJ, 271, 143

Glassgold, A. E., Huggins, P. J., \& Langer, W. D. 1985, ApJ, 290, 615

Hildebrand, R. H. 1983, QJRAS, 24, 267

Hotzel, S., Harju, J., Lemke, D., et al. 2001, A\&A, 372, 302

Hotzel, S. 2001, Ph.D. Thesis, Heidelberg

Hotzel, S., Harju, J., Mattila, D., et al. 2002, in preparation

Juvela, M. 1997, A\&A, 322, 943

Kiss, Tóth, Krause, et al. 2002, in preparation

Kun, M. 1998, ApJS, 115, 59

Larson, R. B. 1981, MNRAS, 194, 809

Launhardt, R., \& Henning, T. 1997, A\&A, 326, 329

Lee, C. W., \& Myers, P. C. 1999, ApJS, 123, 233

Leinert, Ch., Bowyer, S., Haikala, L. K., et al. 1998, A\&AS, 127, 1

Lemke, D., Klaas, U., Abolins, J., et al. 1996, A\&A, 315, L64

Lynds, B. T. 1962, ApJS, 7, 1

Leung, C. M. 1985, in Protostars \& Planets II, ed. D. C. Black, \& M. S. Matthews (The University of Arizona Press, Tucson), 104

Monet, D. G., et al. 1998, USNO-A2.0, Washington DC, USNO

Nelson, R. P., \& Langer, W. D. 1999, ApJ, 524, 923

Stickel, M., Lemke, D., Klaas, U., et al. 2000, A\&A, 359, 865

Tóth, L. V., Hotzel, S., Krause, O., et al. 2000, A\&A, 364, 769

Ward-Thompson, D., André, P., \& Kirk, J. M. 2002, MNRAS, 329, 257

Warin, S., Benayoun, J. J., \& Viala, Y. P. 1996, A\&A, 308, 535

Wheelock, S. L., Gautier, T. N., Chillemi, J., et al. 1994, IRAS sky survey atlas: Explanatory supplement, JPL Publication 94-11, IPAC, JPL

Yonekura, Y., Dobashi, K., Mizuno, A., et al. 1997, ApJS, 110, 21

Yonekura, Y., et al. 2002, priv. comm. 